\title{
Factors Influencing the Evolution of Pulmonary Hypertension in Patients with Hyperthyroidism
}

\author{
CRISTINA TUDORAN, MARIANA TUDORAN*, FLORINA PARV, GHEORGHE NICUSOR POP, AHMED ABU AWWAD, \\ MIHAELA VLAD, MELANIA BALAS \\ University of Medicine and Pharmacy Victor Babes, 2 Eftimie Murgu Sq., 300041, Timisoara, Romania
}

\begin{abstract}
The aim of this study is to document the impact of thyroid hormones on pulmonary hypertension (PH). In two groups of hyperthyroid patients, one with Graves' disease and other with non-autoimmune aetiology, we have determined, by means of echocardiography, the estimated systolic pulmonary artery pressure (PAPS). We have documented an increased incidence of PH and statistically significant correlations between PAPs levels and free thyroxine ( $\left.F T_{A}\right)$, thyroid stimulating hormone (TSH) and TSH receptor antibodies (TRAb) and with the duration of hyperthyroidism. The severity of $\mathrm{PH}$ correlates with the severity and duration of hyperthyroidism. PAPs levels declined gradually, under specific therapy.
\end{abstract}

Keywords: hyperthyroidism, pulmonary hypertension, free thyroxine, thyroid stimulating hormone.

Pulmonary hypertension (PH) is a common cardiovascular complication, diagnosed in patients with hyperthyroidism [1] with an incidence ranging from $24 \%$ [2] to $65 \%$ [3]. According to WHO criteria, $\mathrm{PH}$ is defined by elevated pressures in the pulmonary artery (PAP). According to the European Society of Cardiology's classification, this type of PH is assigned to group 5.3, with unclear and/or multifactorial mechanisms [4]. It has been postulated that PH induced only by hyperthyroidism improves or even achieves normal values after recovering the euthyroid state $[5,6]$.

$\mathrm{PH}$ occurs due to the action of thyroid hormones, thyroxine $\left(T_{4}\right)$ and triiodothyronine $\left(T_{3}\right)$ on the heart and vessels. $T$ is the principal form, acting as a prohormone. Most of $T_{4}$ is converted to biologically active $T_{\text {, through the }}$ removal of an iodide by type 1 deiodinase 77$]$. Thyroid hormones exert their effects on myocytes by genomic and nongenomic mechanisms. Their excess induces tachycardia and increased inotropism, leading to high cardiac output, elevated circulatory volume [8], followed by left ventricular (LV) hypertrophy, diastolic and systolic dysfunction. In the venous territory, there is an enhanced and rapid return to the right ventricle (RV) with pressure overload and RV dilatation, tricuspid regurgitation (TR) and augmentation of PAP and of the pulmonary vascular resistance (PVR). Other data sustain a direct action of thyroid hormones on the pulmonary vasculature and indirect effects on the release of various vasodilators (prostacyclin and nitric oxide) and/or on the metabolism of vasoconstrictors (serotonin, endothelin 1 and thromboxane). In some pathologies (Graves' and Hashimoto disease) there are autoimmune processes, involving thyroid stimulating hormone (TSH) receptor antibodies (TRAb) that could mediate endothelial cells injury [9] and vascular remodelling, leading to aggravation of $\mathrm{PH}[10,11]$. Other factors may also be involved in initiating autoimmune processes $[12,13,18]$.

Evidencing PH could be difficult and assessing mean PAP and PVR implies right heart catheterization, but echocardiography offers the possibility to estimate them accurately without the risks of invasive procedures [14, 15].

The main objective of this study is to document, by means of echocardiography, the incidence and severity of $\mathrm{PH}$ in relation with hyperthyroidism's duration and severity, expressed by the levels of thyroid hormones and of TRAb, in patients with and without Graves' disease with prognostic consequences. Another purpose was to determine the evolution of PAPs at 3,6 and 12 months after specific therapy, when patients have reached the euthyroid status.

\section{Experimental part}

Material and methods

We included in our study 163 patients, 145 women and 18 men, (mean age $50.49 \pm 10.46$ years) with hyperthyroidism of various aetiology, severity and duration, admitted in the Endocrinology Clinic of our hospital between 2013-2017.

To rule out $\mathrm{PH}$ of other aetiology, we have not included in our study patients diagnosed with PH of group 1 or $1^{\prime}$, those with significant cardiovascular pathology that could have produced $\mathrm{PH}$ of group 2, the ones with lungs diseases, causing PH of group 3 and all subjects with history of pulmonary thromboembolism which could have PH group 4. To limit the influence of other factors on the apparition of group $5 \mathrm{PH}$, we excluded all patients with haematological, systemic, metabolic (except hyperthyroidism) or other diseases or chronic renal failure that could induce this form of $\mathrm{PH}$, as well as all subjects treated with vasoactive drugs (calcium channel blocker, alpha-adrenergic blockers, prostacyclin analogues or endothelin receptor antagonists, phospho-diesterase type 5 inhibitors and guanylate cyclase stimulators).

Study groups: depending on the aetiology, subjects were assigned to two groups:

- Group I: patients with Graves' disease - hundred thirtytwo patients with overthyperthyroidism of various severity and duration;

- Group II: patients with non-autoimmune hyperthyroidism included thirty-one patients with overt hyperthyroidism of other aetiology than Graves' disease: multinodular goitre and toxic adenoma of different severity and duration.

Endocrinological assessment: the diagnosis of overt hyperthyroidism was based on the clinical picture and confirmed by the supressed levels of TSH (less than 0.01 $\mathrm{mIU} / \mathrm{L}$ ) and increased levels of free $\mathrm{T}_{4}\left(\mathrm{FT}_{4}\right)$ and/or free $\mathrm{T}_{3}$ $\left(\mathrm{FT}_{3}\right)$. Graves' disease was confirmed by increased titres

*email: mariana.tudoran@gmail.com; Phone: 0040722310302 
of TRAb. Serum TSH, $\mathrm{FT}_{4}$ and $\mathrm{FT}_{3}$ were determined using chemiluminescent microparticle immunoassay (CMIA), normal range being considered as follows: TSH 0.465-4.560 $\mathrm{mIU} / \mathrm{L}, \mathrm{FT}_{4} 0.71-1.85 \mathrm{ng} / \mathrm{mL}$ (9.13-23.81 pmol/L), and $\mathrm{FT}_{3}$ $1.71-3.71^{4} \mathrm{pg} / \mathrm{mL}$ (2.65-5.69 pmol/L). TRÁb were determined by ELISA (Enzyme Linked Immunosorbent Assay), levels higher than $1.75 \mathrm{IU} / \mathrm{mL}$ being considered positive. Thyroid ultrasonography (2D and colour Doppler) was performed using Siemens ultrasound system, with a linear transducer (5.0-14 MHz). In patients with supressed TSH associated with thyroid nodule(s), the diagnosis of toxic adenoma, respectively toxic multinodular goitre was confirmed by $99 \mathrm{mTc}$ thyroid scintigraphy.

Cardiological evaluation: after a rigorous clinical exam and electrocardiography, echocardiographic examinations with an Acuson Sequoia C 512 echocardiograph have been made by the same experienced sonographer, to avoid interobserver differences. After a regular exam of the cardiac morphology and function, we monitored echocardiographically, the following parameters by measuring them at the initial visit and at 3, 6 and 12 months of therapy when patients achieved euthyroidism.

1.Right atrial (RA), RV diameter and TR velocity (TRV);

2.Estimated systolic PAP (PAPs); in this study we considered that PAPs values $\geq 35 \mathrm{mmHg}$, indicates $\mathrm{PH}$ ( 3 , 10) with severity ranging from mild $(35-49 \mathrm{mmHg})$, to moderate $(50-69 \mathrm{mmHg})$ and severe $(\leq 70 \mathrm{mmHg})[14,15]$.

3.Estimated Pulmonary Vascular Resistance (PVR); we considered as elevated PVR $\leq 2 \mathrm{WU}$ and significant $\mathrm{PH}$ was defined by PVR $>3$ WU [14].

Data analysis was performed using SPSS v. 24.0 (Statistical Package for the Social Sciences, Chicago, IL, USA). Continuous variables were presented as a mean and standard deviation (SD) or median and associated quartiles (Q1-25 percentage quartile, Q3-75 percentage quartile) and categorical data were presented as counts (percentages). The bias-corrected and accelerated (BCa) bootstrap interval (1000 bootstrap samples) was used to calculate the $95 \%$ confidence interval. We performed descriptive and inferential statistics analysis to summarize the characteristics of the study population. To evaluate the proportion of pulmonary hypertension in groups, we applied the chi-squared test $(\chi 2)$. The results of the Shapiro-Wilk normality test showed a non-Gaussian distribution, which is why we continued to use nonparametric tests. In order to highlight the factors influencing PH's evolution, we analysed the strength of a linear relationship between PAPs, $\mathrm{PVR}, \mathrm{FT}_{4}$ and the duration of hyperthyroidism by using the Spearman's rank-order correlation. To compare patients with/without PH, characteristics in table 1 we applied Mann-Whitney $U$ test. Friedman test followed by a posthoc analysis with Wilcoxon signed-rank tests was conducted with a Bonferroni correction applied, in order to evaluate the evolution of PAPs in our groups over a period of twelve months. A p value of less than 0.05 was considered to indicate a statistically significance.The study was approved by the Ethics Committee of our hospital and all patients signed a written consent.

\section{Results and discussions}

Our study included 163 patients, aged between 23 and 70 years with overt hyperthyroidism of various aetiology. The majority had Graves' disease 132 (80.98\%) and 31 (19.01\%) had hyperthyroidism of non-autoimmune aetiology: multinodular goitre (20 - 64.51\%) and toxic adenoma (11 - 35.48\%). This distribution is characteristic for our geographic region, with a high prevalence of Graves' disease. This pathology was eleven times more frequent in women than in men. Regarding associated diseases, none of our patients had pathological conditions inducing $\mathrm{PH}$.

In our study, we have determined PAPs by Doppler echocardiography which offers a reliable estimation of PAPs $[14,15]$. There are conflicting data in the literature regarding the strength of the correlation between the PAPs assessed by this method and mean PAP measured via right heart catheterization. Despite its diagnostic accuracy the last method is invasive, with potentially serious risks, not being justified solely for screening purposes.

We documented in our study group a high incidence of PH (53.37\%). Similarly, Armigliato reported a prevalence of $65 \%$, Marvisi of $43 \%$ and Jonathan $\mathrm{H}$. Li one of $24 \%$ in studies of patients with newly diagnosed hyperthyroidism, by using Doppler echocardiography $[3,5,10]$. The higher incidence found in our study can be explained by the fact that we included only hospitalised patients, many of them with severe forms. In our study, patients with Graves' disease had a higher incidence of $\mathrm{PH}$ than nonautoimmune hyperthyroidism (54.54\%, versus $48.38 \%$ ), sustaining the hypothesis that autoimmune processes, associated with endothelial damages, may play a major role in the pathogenesis of $\mathrm{PH}$. This aspect was mentioned in other articles [1, 3]. PH was more frequent in women, the influence of female hormonal status in various cardiovascular pathologies being debated also in other studies [16]. Due to the fact that in our study, were included only fifteen males, the statistical analysis was not conclusive in detecting gender related differences regarding $\mathrm{PH}$.

Referring to the severity of hyperthyroidism, there were 46 patients with severe thyrotoxicosis. The duration of the disease was another criterion followed in our study. There were 39 patients with overt hyperthyroidism, diagnosed for more than 2 years, with several relapses, who have refused/delayed surgery or therapy with radioactive iodine and were treated only with oral antithyroid drugs.

The results of clinical, laboratory and echocardiographic examinations are presented in table 1 and median values of PAPs and PVR in patients from group I and II, with and without $\mathrm{PH}$, at the initial visit, are illustrated in figure 1.

Thyroid dysfunction was treated according to aetiology and severity: either surgery or therapy with radioactive iodine or oral antithyroid drugs in order to restore euthyroidism as soon as possible. Most patients received beta-blocker, in order to treat cardiovascular symptoms: tachycardia, arrhythmias or elevated blood pressure values.

In group I, with Graves' disease, there were 121 women (91.66\%) and 11 men (8.33\%), aged between 23 and 63 years. 72 patients $(54.54 \%)$ had PH of various severity, median of PAPs $=61.5(40.25-74) \mathrm{mmHg}$ and median of $\mathrm{PVR}=3.8(3.8-5.3) \mathrm{WU} ; 24$ patients (33.33\%) had severe $\mathrm{PH}, 22(30.55 \%)$ moderate and $26(36.11 \%)$ mild forms. Regarding the gender distribution, from all patients with $\mathrm{PH} 68(94.44 \%)$ were women. For patients with $\mathrm{PH}$ we emphasized a strong positive correlation between PAPs and $\mathrm{FT}_{4}$ levels $(r=0.889, p . . .0 .001,95 \% \mathrm{Cl}[0.835 ; 0.920]$, a moderate one with PVR ( $r=0.697, p \ldots . . .0 .001,95 \% \mathrm{Cl}$ [ 0.504 ; $0.837]$, with the duration of the disease, $(r=0.685, p . . .0 .001$, $95 \% \mathrm{Cl}[0.526 ; 0.798])$, with TRAb level, $r=0.595, p \ldots . . .0 .001$, $95 \% \mathrm{Cl}[0.412 ; 0.741]$ and a moderate negative one with TSH, r $=-0.498$, p..0.001, 95\%Cl [-0.679; - 0.3], (fig. 2).

Referring to 31 patients with non-autoimmune hyperthyroidism, 24 women $(77.41 \%)$ and 7 men $(22.58 \%)$, aged betw een 35 to 70 years. 16 patients had no $\mathrm{PH}$, but in $15(48.38 \%), 12$ women (80\%) and 3 men (20\%), we have documented $\mathrm{PH}$, with a median PAPs of $49(43-69) \mathrm{mmHg}$ 
Table 1

CHARACTERISTICS AND ECHOCARDIOGRAPHIC RESULTS IN THE STUDY GROUPS

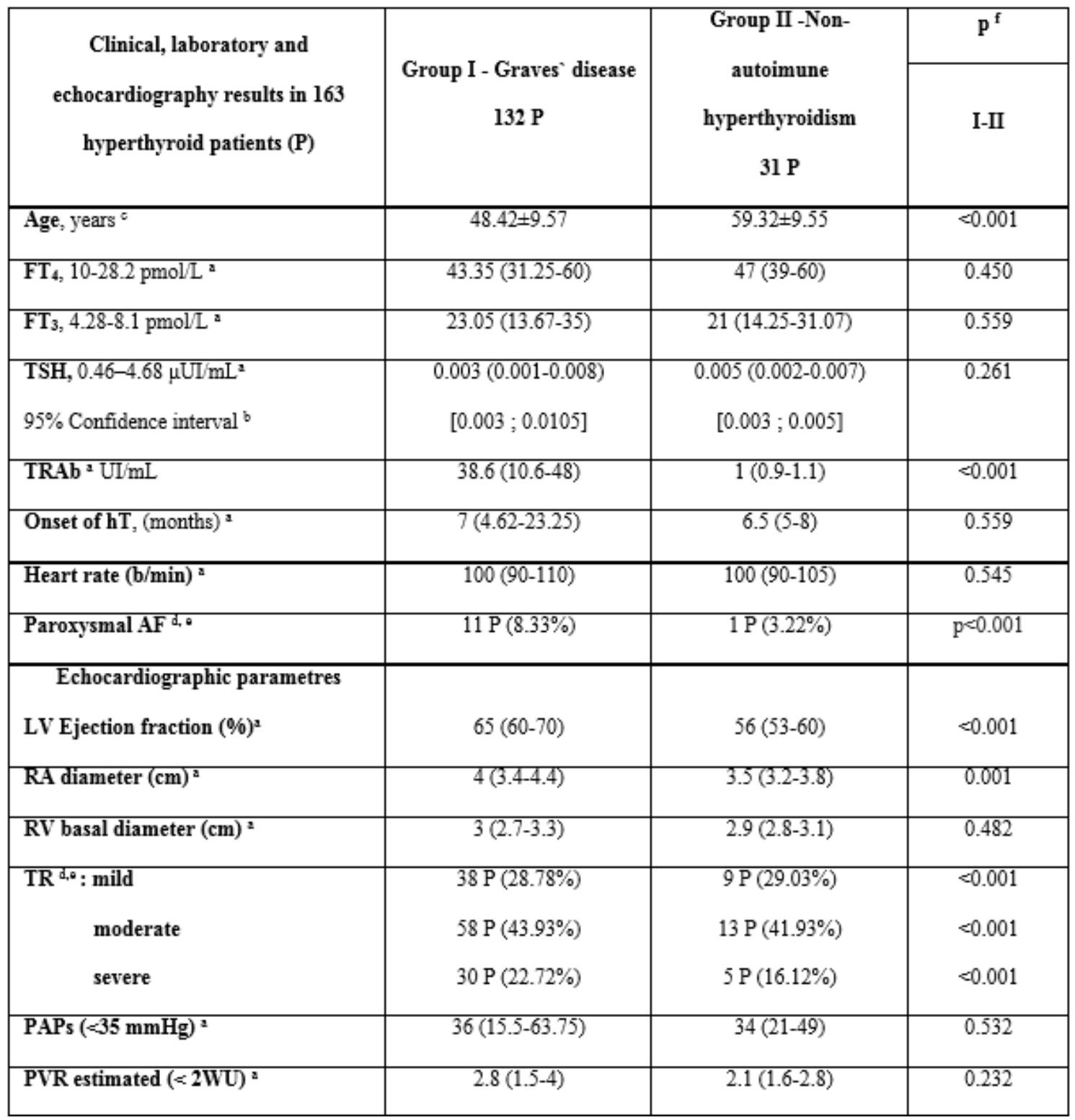

Legend: $F T_{4}=$ free thyroxin; $F T_{3}=$ free triiodothyronine; $T S H=$ thyroid stimulating hormone; $T R A b=T S H$ receptor antibodies; $A F=$ atrial fibrillation; $L V=$ left ventricle; $R V=$ right ventricle; $R A=$ right atria; $T R=$ tricuspid regurgitation; $P A P s=$ estimated systolic pressure in the pulmonary artery; PVR=pulmonary vascular resistance; WU=Wood Units. a. median and associated quartiles (Q1 - Q3); b. The bias-corrected and accelerated (BCa) bootstrap interval (1000 bootstrap samples); c. mean and standard deviation (SD); d. counts (percentages); e. Chi-Square test; f. Mann-Whitney U Test
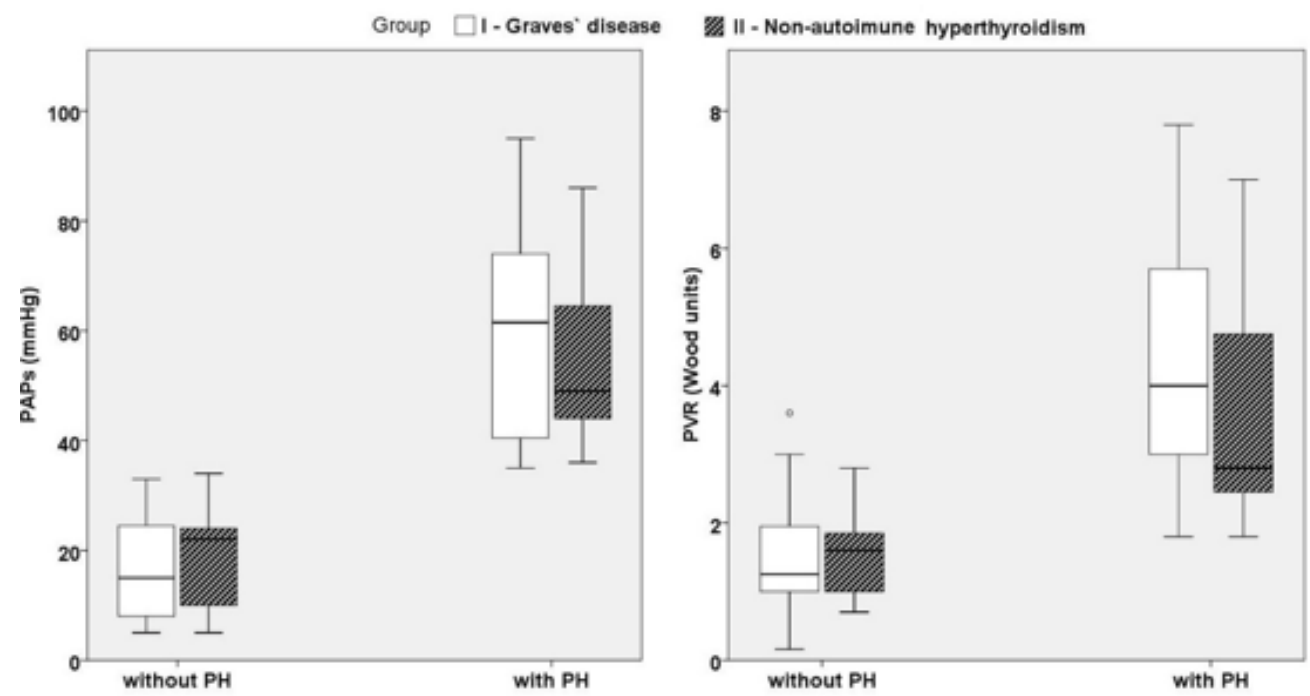

Fig. 1 Distributions of PAPs and PVR levels in group I and II

Legend: PAPs=estimated pulmonary artery pressure; PVR=estimated pulmonary vascular resistance; $\mathrm{PH}=$ pulmonary hypertension. Whitney Mann U-test. 


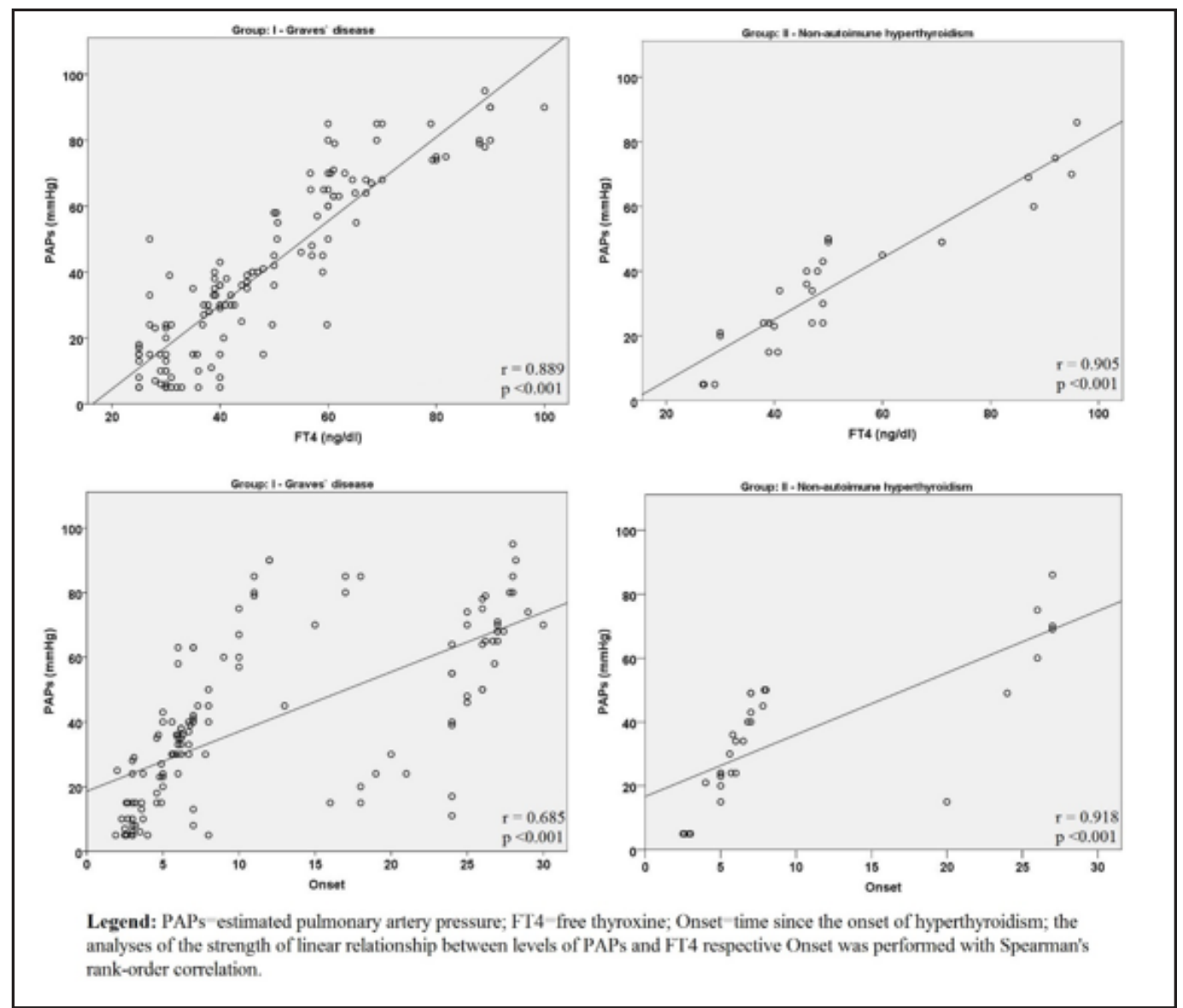

Fig. 2. Correlations between PAPs and $\mathrm{FT}_{4}$, respective onset in group I and II
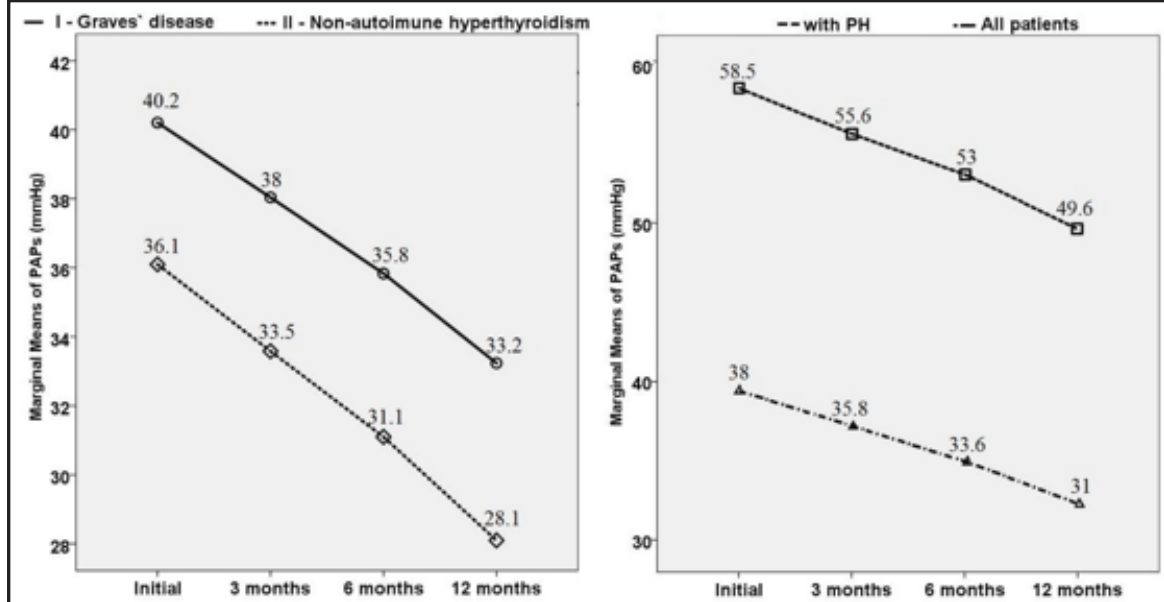

Legend: PAPs=pulmonary arterial pressure; the Friedman test followed by a post-hoc analysis with Wilcoxon signed-rank tests was conducted with a Bonferroni correction applied resulting in a significance level set at $\mathrm{p}<0.008$.

Fig. 3. Evolution of PAPs at 3, 6 and 12 months in patients with and without $\mathrm{PH}$

and a median PVR of 2.8 (2.25-4.37) WU. As severity, 3 (20\%) had severe $\mathrm{PH}, 4$ (26.66\%) moderate and $8(53.33 \%)$ mild forms. For these patients with $\mathrm{PH}$, we have documented a strong positive, correlation between PAPs and $\mathrm{FT}_{4}$ levels, $\mathrm{r}=0.905$, p....0.001, $95 \% \mathrm{Cl}[0.616 ; 0.986]$, with the duration of the disease, $r=0.918, p \hat{A} 0.001,95 \% \mathrm{Cl}$ [0.783; 0.974] and with PVR, $r=0.747, p=0.001,95 \% \mathrm{Cl}$ [0.212; 0.986], (fig. 2). With TSH levels we found a strong negative correlation, $r=-0.737, p=0.002,95 \% \mathrm{Cl}[-0.951 ;$ $0.323]$.

We followed the evolution of PAPs levels at 3, 6 and 12 months. The Friedman test demonstrated a statistically significant difference for both groups $(p<0.001)$. Post-hoc analysis with Wilcoxon signed-rank tests and Bonferroni correction applied, set significance level at $p<0.008$, have shown a significant reduction of PAPs at twelve months for patients with Graves' disease and with non-autoimmune hyperthyroidism ( $Z=-2.62$, respective, $Z=-2.56$, $p<0.001)$. Reporting to the initial levels of PAPs, we noticed in all patients a significant reduction every 3 months, leading to a decrease of $22.9 \%$ at 12 months $(Z=-2.61$, p<0.001) (fig. 3).

It is possible that the duration of follow-up for the group with persistent disease was too small to draw any significant conclusions. Some authors followed the incidence and evolution of PH by Doppler echocardiography initially and after antithyroid treatment. These hemodynamic abnormalities were reversible in the majority of patients with $\mathrm{PH}$ after restoration of the euthyroid state $[5,6]$. 
As our knowledge on the pathophysiology of $\mathrm{PH}$ progresses, it is obvious that a comprehensive approach to the pulmonary circulation and RV's physiology, will be beneficial for the clinical management of $\mathrm{PH}$ patients and scientific research [17].

\section{Conclusions}

In our study we have documented that PH was more frequent in female patients with Graves' disease and its gravity correlates with the severity and duration of hyperthyroidism. PAPs values declined gradually, under specific therapy. In severe and recurrent cases, the reduction of PAPS was not so significant.

\section{References}

1. BIONDI B, KAHALY GJ , Cardiovascular involvement in patients with different causes of hyperthyroidism; nature reviews endocrinology, nr. 6, 2010, p. 431.

2. LI JH, SAFFORD RE, ADUEN JF, HECKMAN MG, CROOK JE, BURGER $C D$, Pulmonary hypertension and thyroid disease. chest, 132, 2007, $p$. 793.

3. ARMIGLIATO M, PAOLINI R, AGGIO S, ET AL. Hyperthyroidism as a cause of pulmonary arterial hypertension: prospective study, angiology, 57, nr.5, 2006, p. 600.

4. NAZZARENO GM, SIMON-GIBBSI, LANG A, TORBICKI G, SIMONNEAU A, PEACOCK A, ET ALL, 2015 esc/ers Guidelines for the diagnosis and treatment of pulmonary hypertension: the joint task force for the diagnosis and treatment of pulmonary hypertension of the european society of cardiology and the european respiratory society, european heart journal, 37, nr.1, 2016 p. 67.

5. MARVISI M., ZAMBRELLI P., BRIANTI M., CIVARDI G., LAMPUGNANI R., DELSIGNORE R., Pulmonary hypertension is frequent in hyperthyroidism and normalizes after therapy, eur $\mathrm{j}$ intern med., 17, 2006, p. 267.

6. MARMOUCH H, FADIA BOUBAKER, SONDES ARFA, TANSIM SLIM, HANENE SAYADI, MANEL J MAL \& INES KHOCHTALI - Transient pulmonary hypertension in patients with graves' disease endocrine abstracts 37, 2015, p.936.

7. MEJIA OSUNA P, UDOVCIC M, SHARMA MD, Hyperthyroidism and the heart, houston methodist hospital, houston, mdcvj, 13, nr. 2 , 2017, p. 60 .
8. JABBAR A., PINGITORE A., PEARCE S.H.S., ZAMAN A., IERVASI G., RAZVI S., Thyroid hormones and cardiovascular disease, nature reviews cardiology, 14, 2017, p. 39.

9. MARVISI M., BALZARINI L., MANCINI C., MOUZAKITI P., Thyroid gland and pulmonary hypertension, what's the link? panminerva med. 55, nr.1, 2013 p. 93.1

0. LI J.H, SAFFORD RE, ADUEN JF, HECKMAN MG ET ALL, Pulmonary hypertension and thyroid disease, chest, 132, 2007, p. 793.

11. SCICCHITANO P, DENTAMARO I, TUNZI F, RICCI G, CARBONARA $\mathrm{S}$, et. all., Pulmonary hypertension in thyroid diseases, endocrine, 19, nr. 3, 2016 p. 578.

12. MIHAI, C., CIJEVSCHI PRELIPCEAN, C., DRANGA, M., et all., Correlations between inflammatory biomarkers and activity in inflammatory bowel diseases, Rev. Chim. (Bucharest), 69, no. 3, 2018, p. 710 .

13. COCOLOS, A.M., DUMITRU, N., NEDELTCHEVA PETROVA E., COCOLOS, I., TIGLIS, M., ET ALL, Endocrine disrupting chemicals the $x$ factor in different pathologies, Rev. Chim. (Bucharest), 69, no.1, 2018, p. 134.

14. ABBAS AE, FRANEY LM, MARWICK T, MAEDER MT, KAYE DM, VLAHOS AP ET ALL, Pulmonary vascular resistance by doppler echocardiography, jase, 26, nr. 10, 2013 p. 1170.

15. NAING P, KUPPUSAMY H, SCALIA G, et. all., Non-invasive assessment of pulmonary vascular resistance in pulmonary hypertension: current knowledge and future direction. heart, lungs and circulation, 26, nr. 4, 2017, p. 323.

16. ANGHEL, L., PRISACARIU, C., ARSENESCU GEORGESCU, C., Is there a sex difference of cardiovascular risk factors in patients with acute myocardial infarction? Rev. Chim. (Bucharest), 69, no. 1, 2018, p. 255.

17. BADANO L.P., GINGHINA C., EASAW J., MURARU D., GRILLO M.T., LANCELLOTTI P., ET ALL - Night ventricle in pulmonary arterial hypertension: haemodynamics, structural changes, imaging, and proposal of a study protocol aimed to assess remodelling and treatment effects. eur $\mathrm{j}$ echocardiogr nr. 11, 2010, p. 27.

18. ABU AWWAD AHMED, PREJ BEANU, R., VERMESAN, D., BRANEA, I., DELEANU, B., FLORESCU, D., VLAD-DALIBORCA, C., Blood Loss Of Pedicle Subtraction Osteotomy For Sagittal Imbalance Spinal Deformity, no 12,2018, p. 3680

Manuscript received: 6.09 .2018 